\title{
On positive periodic solutions of second order singular equations
}

\author{
Yunhai Wang ${ }^{1}$ and Yuanfang $\mathrm{Ru}^{2^{*}}$
}

\author{
"Correspondence: \\ ruyanfangmm@163.com \\ ${ }^{2}$ College of Science, China \\ pharmaceutical University, Nanjing, \\ P.R. China \\ Full list of author information is \\ available at the end of the article
}

\begin{abstract}
Using the fixed point theorem, we study the existence and multiplicity of positive periodic solutions for the second order differential equations

$$
\left\{\begin{array}{l}
\ddot{x}+a(t) x=f(x), \\
x(0)=x(T), \quad \dot{x}(0)=\dot{x}(T) .
\end{array}\right.
$$

For given nonnegative constants $0<\beta_{1}<\beta_{2}<\cdots<\beta_{N}$, the function $f$ may be singular at $x=\beta_{i}$.
\end{abstract}

Keywords: Periodic solutions; Second order differential equations; Singular; Fixed point theorem

\section{Introduction}

We say that the differential equation

$$
\ddot{x}+a(t) x=\lambda f(t, x)+e(t)
$$

is singular if the nonlinear term $f$ is singular, which means that a vector-valued function $f$ is only defined on $\mathbb{R} \times \mathbb{R}^{N} \backslash \Omega$ with $\Omega \subset \mathbb{R}^{N}$ being nonempty and for any $x_{0} \in \Omega$,

$$
\lim _{x \rightarrow x_{0}} f(t, x)=+\infty, \quad \text { uniformly in } t \in \mathbb{R} \text {. }
$$

During the last two decades, the existence of nontrivial periodic solutions of (1.1) has been studied by many researchers in nonsingular case as well as singular case. Usually, the proof is based on either the method of upper and lower solutions [2, 10, 15], fixed point theorems $[6,7,17-19]$, alternative principle of Leray-Schauder $[4,11]$, or topological degree theory $[23,24]$. To our attention, Wang in [22] studied the existence, multiplicity, and nonexistence results for positive solutions of the singular periodic boundary value problem (1.1) in terms of different values of $\lambda \in \mathbb{R}$. But most of these papers are concerned with singularity at $x=x_{0}$. Later, a similar idea is used to study the singular periodic systems with two parameters,

$$
\left\{\begin{array}{l}
\ddot{u}+a_{1}(t) u=\lambda b_{1}(t) f_{1}(u, v), \\
\ddot{v}+a_{2}(t) v=\mu b_{2}(t) f_{2}(u, v),
\end{array}\right.
$$

(c) The Author(s) 2018. This article is distributed under the terms of the Creative Commons Attribution 4.0 International License (http://creativecommons.org/licenses/by/4.0/), which permits unrestricted use, distribution, and reproduction in any medium, provided you give appropriate credit to the original author(s) and the source, provide a link to the Creative Commons license, and indicate if changes were made. 
where $(\lambda, \mu) \in \mathbb{R}_{+}^{2} \backslash\{0,0\}$. One nice result proved in [20] is that there exist three nonempty subsets of $\mathbb{R}_{+}^{2} \backslash\{0,0\}: \Gamma, \Delta_{1}, \Delta_{2}$ such that $\mathbb{R}_{+}^{2} \backslash\{0,0\}=\Gamma \cup \Delta_{1} \cup \Delta_{2}$ and (1.2) has at least two positive periodic solutions for $(\lambda, \mu) \in \Delta_{1}$, one positive periodic solution for $(\lambda, \mu) \in$ $\Gamma$, and no positive periodic solutions for $(\lambda, \mu) \in \Delta_{2}$. Note that in this paper the word "singularity" is understood in a more general way because we only need that $f_{1}$ is singular at the whole line $x$-axis and $f_{2}$ is singular at the whole line $y$-axis. The proof is based on the following vector version of Krasnosel'skii's fixed point theorem [14]. In addition, there are many papers concerned with the existence and multiplicity of radial solutions of the elliptic equations with the regular or singular nonlinearities. We refer the readers to $[1,3$, $5,8,12,13,16,21]$.

Motivated by these recent developments, in this paper, we investigate the existence and multiplicity of $T$-periodic solutions of the following problem:

$$
\left\{\begin{array}{l}
\ddot{x}+a(t) x=f(x), \\
x(0)=x(T), \quad \dot{x}(0)=\dot{x}(T),
\end{array}\right.
$$

where the function $f$ may be singular at $x=\beta_{i}(i=1, \ldots, N)$. For convenience, we give the following notations:

$$
\begin{aligned}
& a_{*}=\min _{t \in[0, T]} a(t), \quad a^{*}=\max _{t \in[0, T]} a(t), \\
& f^{*}(r)=\max \{f(x): \theta r \leq x \leq r\}, \quad \text { where } r>0, \theta \in(0,1) .
\end{aligned}
$$

For $v=0$ or $v=+\infty$, there exist nonnegative constants $f^{v}$ defined as

$$
f^{v}=\lim _{x \rightarrow v} \frac{f(x)}{x}
$$

The main results can be expressed as follows.

Theorem 1.1 Assume that the function $f$ satisfies the following conditions:

(A) $f$ may be singular at $x=\beta_{i}(i=1, \ldots, N)$ and continuous on

$$
\left(0, \beta_{1},\right),\left(\beta_{1}, \beta_{2}\right), \ldots,\left(\beta_{N-1}, \beta_{N}\right),\left(\beta_{N},+\infty\right)
$$

(B) For any interval $\left(\beta_{i}, \beta_{i+1}\right), i=1, \ldots, N-1$,

(i) $f(x)-a^{*} \beta_{i} \geq 0, \quad$ for $x \in\left(\beta_{i}, \beta_{i+1}\right)$,

(ii) there exists $\bar{R}_{i} \in\left(\beta_{i}, \beta_{i+1}\right)$ such that

$$
\max _{x \in\left[\theta \bar{R}_{i}+(1-\theta) \beta_{i}, \bar{R}_{i}\right]} f(x)<\frac{\bar{R}_{i}-\beta_{i}}{M T}+a_{*} \beta_{i} .
$$

Then (1.3) has at least $(2 N-2)$ positive periodic solutions.

Theorem 1.2 Assume that (A) and (B) hold. In addition, the function $f$ satisfies the following condition: 
(C) There exists $\bar{R}>\beta_{N}$ such that

(i) $f(x)-a^{*} \beta_{N} \geq 0, \quad$ for $x \in\left(\beta_{N}, \bar{R}\right)$,

(ii) $\max _{x \in\left[\theta \bar{R}+(1-\theta) \beta_{N}, \bar{R}\right]} f(x)<\frac{\bar{R}-\beta_{N}}{M T}+a_{*} \beta_{n}$.

Then (1.3) has at least $(2 N-1)$ positive periodic solutions.

Theorem 1.3 Assume that (A) and (B) hold. In addition, the function $f$ satisfies the following condition:

(D) There exists $\bar{r} \in\left(0, \beta_{1}\right)$ such that $f^{*}(\bar{r})<\frac{\bar{r}}{M T}$.

Then (1.3) has at least $(2 N-1)$ positive periodic solutions.

Corollary 1.4 Assume that (A) and (B) hold. If $f^{0}=0$, then (1.3) has at least $(2 N-1)$ positive periodic solutions.

Theorem 1.5 Assume that (A), (B), and (D) hold. If $f^{0}=+\infty$, then (1.3) has at least $(2 N)$ positive periodic solutions.

Theorem 1.6 Assume that (A), (B), (C), and (D) hold. Then (1.3) has at least (2N) positive periodic solutions.

Theorem 1.7 Assume that (A) and (B) hold. In addition, the function $f$ satisfies the following condition:

(E) There exists $\hat{R}>\frac{\beta_{N}}{\theta}$ such that

(i) $f(x)-a^{*} \beta_{N} \geq 0, \quad$ for $x \in\left(\beta_{N}, \hat{R}\right)$,

(ii) $\max _{x \in[\theta \hat{R}, \hat{R}]} f(x)<\frac{\bar{R}}{M T}+\min \left\{a_{*}-\frac{1}{M T}, 0\right\} \beta_{N}$.

If $f^{\infty}=+\infty$, then (1.3) has at least $(2 N)$ positive periodic solutions.

Theorem 1.8 Assume that (A), (B), (C), and (D) hold. If $f^{0}=+\infty$, then (1.3) has at least $(2 N+1)$ positive periodic solutions.

Theorem 1.9 Assume that (A), (B), (D), and (E) hold. If $f^{\infty}=+\infty$, then (1.3) has at least $(2 N+1)$ positive periodic solutions.

Theorem 1.10 Assume that (A), (B), (D), and (E) hold. If $f^{0}=+\infty$ and $f^{\infty}=+\infty$, then (1.3) has at least $(2 N+2)$ positive periodic solutions.

\section{Preliminary}

The function $a$ satisfies the following assumption:

(H) The function $a$ is continuous, positive, $T$-periodic and the linear equation $\ddot{x}+a(t) x=0$ has a positive Green's function $G(t, s)$, i.e.,

$$
\begin{aligned}
& G(t, s)>0 \quad \text { for all }(t, s) \in[0, T] \times[0, T], \\
& m=\min _{0 \leq s, t \leq T} G(t, s)>0, \quad M=\max _{0 \leq s, t \leq T} G(t, s), \quad \theta=m / M \in(0,1) .
\end{aligned}
$$


From $(\mathrm{H})$, it is clear that a function $x(t)$ is a $T$-periodic solution of (1.3) if and only if

$$
x(t)=\int_{0}^{T} G(t, s) f(x(s)) d s
$$

Define the operator $A: K \rightarrow E$ by

$$
A x(t)=\int_{0}^{T} G(t, s) f(x(s)) d s, \quad 0 \leq t \leq T .
$$

Let $E$ denote the Banach space $C[0, T]$ with the usual max-norm and define a subcone $K$ by

$$
K=\{x(t) \in C[0, T]: x(t) \geq \theta\|x\|\}
$$

For $r>0$, let

$$
\begin{aligned}
& K_{r}:=\{x(t) \in K:\|x(t)\|<r\}, \\
& \partial K_{r}:=\{x(t) \in K:\|x(t)\|=r\} .
\end{aligned}
$$

Therefore, the solution of (1.3) is equivalent to the fixed point of the operator $A$. The discussion is based on the following well-known fixed point theorem.

Lemma 2.1 ([9, Theorem 2.3.4]) Let $E$ be a Banach space and $K \subset E$ be a cone in $E$. Assume that $\Omega_{1}, \Omega_{2}$ are open subsets of $E$ with $0 \in \Omega_{1}, \bar{\Omega}_{1} \subset \Omega_{2}$, and let $T: K \cap\left(\bar{\Omega}_{2} \backslash \Omega_{1}\right) \rightarrow$ $K$ be a completely continuous operator such that either

(i) $\|T u\| \leq\|u\|, u \in K \cap \partial \Omega_{1}$ and $\|T u\| \geq\|u\|, u \in K \cap \partial \Omega_{2}$; or

(ii) $\|T u\| \geq\|u\|, u \in K \cap \partial \Omega_{1}$ and $\|T u\| \leq\|u\|, u \in K \cap \partial \Omega_{2}$.

Then $T$ has a fixed point in $K \cap\left(\bar{\Omega}_{2} \backslash \Omega_{1}\right)$.

\section{Proof of the main results}

First, we consider the existence of solutions of (1.3) on $\left(0, \beta_{1}\right)$.

Lemma 3.1 Assume that (H), (A), and (D) hold. Then (1.3) has at least one positive periodic solution.

Proof Since $f(\cdot)$ is singular at $x=\beta_{1}^{-}$, there exists sufficiently small $\delta<(1-\theta) \beta_{1}$ such that $f(x) \geq \rho x$, for $0<\left|x-\beta_{1}\right|<\delta_{1}$, where $\rho$ satisfies $\rho m\left|\Omega_{2}\right| \theta \geq 1, \Omega_{2}$ is given as follows. Choose $R \in\left(\beta_{1}-\delta, \beta_{1}\right)$. Then, for $x \in \partial K_{R}$, one has $x(t) \geq \theta\|x\| \geq \theta R$. If $\theta R \geq \beta_{1}-\delta$, then $\frac{\beta_{1}-\delta}{\theta} \leq R \leq \beta_{1}$. Further, we have $\delta>(1-\theta) \beta_{1}$, which yields a contradiction. Now we only consider the case $\theta R<\beta_{1}-\delta$. Let $[0, T]=\Omega_{1} \cup \Omega_{2}$, where

$$
\begin{aligned}
& \Omega_{1}=\left\{t \in[0, T]: \theta R \leq x(t)<\beta_{1}-\delta\right\} \\
& \Omega_{2}=\left\{t \in[0, T]: \beta_{1}-\delta \leq x(t) \leq R\right\} .
\end{aligned}
$$


Since $\|x\|=R$ and $x$ is continuous, $\Omega_{2}$ is nonempty and $\left|\Omega_{2}\right|>0$. Therefore, we have

$$
\begin{aligned}
\|A x\| & =\max _{t \in[0, T]} \int_{0}^{T} G(t, s) f(x(s)) d s \\
& \geq m\left[\int_{\Omega_{1}} f(x(s)) d s+\int_{\Omega_{2}} f(x(s)) d s\right] \\
& \geq m \int_{\Omega_{2}} f(x(s)) d s \geq \rho m\left|\Omega_{2}\right| \theta\|x\| \geq\|x\| .
\end{aligned}
$$

For any $x \in \partial K_{\bar{r}}$, we have

$$
\|A x\|=\max _{t \in[0, T]} \int_{0}^{T} G(t, s) f(x(s)) d s \leq \int_{0}^{T} G(t, s) \frac{\bar{r}}{M T} d s<\|x\| .
$$

From [22], it is clear that $A: \overline{K_{R}} \backslash K_{\bar{r}} \rightarrow K$, and $A$ is completely continuous on $\overline{K_{R}} \backslash K_{\bar{r}}$. Therefore, by Lemma 2.1, (1.3) has at least a positive solution $x(t) \in \overline{K_{R}} \backslash K_{\bar{r}}$.

Lemma 3.2 Assume that $(\mathrm{H})$ and $(\mathrm{A})$ hold. If $f^{0}=0$, then (1.3) has at least one positive periodic solution.

Proof Since $f^{0}=0$, there exists sufficiently small $\bar{r}>0$ such that $f(x) \leq \epsilon x$ for $x \in[0, \bar{r}]$, where $\epsilon M T<1$. Then, for any $x \in \partial K_{\bar{r}}$, we have

$$
\|A x\|=\max _{t \in[0, T]} \int_{0}^{T} G(t, s) f(x(s)) d s \leq \epsilon M T\|x\|<\|x\| .
$$

The remainder is similar to the proof of Lemma 3.1, so we omit it.

Lemma 3.3 Assume that (H), (A), and (D) hold. If $f^{0}=+\infty$, then (1.3) has at least two positive periodic solutions.

Remark $f^{0}=+\infty$ implies that $f$ may be singular at $x=0$.

Proof Since $f(\cdot)$ is singular at $x=\beta_{1}^{-}$, from the proof of Lemma 3.1, there exists $R \in\left(\beta_{1}-\right.$ $\left.\delta, \beta_{1}\right)>\bar{r}$ such that $\|A x\|>\|x\|$ for $x \in \partial K_{R}$

Since $f^{0}=+\infty$, there exists sufficiently small $r<\bar{r}$ such that $f(x) \geq \varrho x$ for $x \in[0, r]$, where $\varrho m \theta T>1$. Then, for any $x \in \partial K_{r}$, we have

$$
\|A x\|=\max _{t \in[0, T]} \int_{0}^{T} G(t, s) f(x(s)) d s \geq \varrho m \theta T\|x\|<\|x\| .
$$

For any $x \in \partial K_{\bar{r}}$, we have

$$
\|A x\|=\max _{t \in[0, T]} \int_{0}^{T} G(t, s) f(x(s)) d s \leq \epsilon M T\|x\|<\|x\| .
$$

Further, from (A) and the Arzela-Ascoli theorem it follows that now we verify that $A$ : $\overline{K_{R}} \backslash K_{r} \rightarrow K$, and $A$ is completely continuous. 
Therefore, by Lemma 2.1, (1.3) has at least two positive solutions $x_{1}(t) \in \overline{K_{R}} \backslash K_{\bar{r}}$ and $x_{2}(t) \in \overline{K_{\bar{r}}} \backslash K_{r}$.

Second, we give some existence results of solutions of (1.3) on $\left(\beta_{N},+\infty\right)$.

Lemma 3.4 Assume that (H), (A), and (C) hold. Then (1.3) has at least one positive periodic solution.

Remark Since $f(\cdot)$ is singular at $x=\beta_{N}^{+}$, there exists sufficiently small $\delta<\frac{1-\theta}{\theta}$ such that $f(x) \geq \rho x$ for $0<x-\beta_{N}<\delta$. Choose $r \in\left(\beta_{N}, \beta_{N}+\delta\right)$. Then, for $x \in \partial K_{r}$, one has $x(t) \geq$ $\theta\|x\| \geq \theta r$. If $\theta r \geq \beta_{n}$, then $\frac{\beta_{N}}{\theta} \leq r \leq \beta_{N}+\delta$, which yields a contradiction. Therefore $\theta r<$ $\beta_{N}$, which implies that $A$ is not continuous on $\overline{K_{R}} \backslash K_{r}$, where $R>\frac{\beta_{N}}{\theta}$.

Proof Let $\omega=u-\beta_{N}$. Then (1.3) is equivalent to the problem

$$
\left\{\begin{array}{l}
\ddot{\omega}+a(t) \omega=f\left(\omega+\beta_{N}\right)-a(t) \beta_{N}=F(\omega), \\
\omega(0)=\omega(T), \quad \dot{\omega}(0)=\dot{\omega}(T) .
\end{array}\right.
$$

From (A) and (C), it follows that

$\left(a_{1}\right) \lim _{\omega \rightarrow 0^{+}} F(\omega)=+\infty$;

$\left(a_{2}\right)$ let $\widetilde{r}=\bar{R}-\beta_{N}, F(\omega) \geq 0$, for $\omega \in(0, \widetilde{r})$;

$\left(a_{3}\right)$ there exists sufficiently small $\bar{r}<\tilde{r}$ such that

$$
F(\omega)>L\|\omega\|, \quad \text { for } \omega \in(0, \bar{r})
$$

where $\operatorname{LmT}>1$.

Define the operator $A: K \rightarrow E$ by

$$
T x(t)=\int_{0}^{T} G(t, s)\left[f\left(\omega(s)+\beta_{N}\right)-a(t) \beta_{N}\right] d s, \quad 0 \leq t \leq T .
$$

Then, for any $\omega \in \partial K_{\widetilde{r}}$, from (ii) of (C), we have

$$
\begin{aligned}
\max _{\omega \in[\theta \tilde{r}, \tilde{r}]}\left[f\left(\omega(s)+\beta_{N}\right)-a(t) \beta_{N}\right] & \leq \max _{\omega \in[\theta \tilde{r}, \tilde{r}]}\left[f\left(\omega(s)+\beta_{N}\right)-a_{*} \beta_{N}\right] \\
& =\max _{x \in\left[\theta \bar{R}+(1-\theta) \beta_{N}, \bar{R}\right]} f(x)-a_{*} \beta_{N}<\frac{\tilde{r}}{M T} .
\end{aligned}
$$

Further, we have

$$
\begin{aligned}
\|T \omega\| & =\max _{t \in[0, T]} T \omega(t)=\max _{t \in[0, T]} \int_{0}^{T} G(t, s)\left[f\left(\omega(s)+\beta_{N}\right)-a(t) \beta_{N}\right] d s \\
& \leq M T \max _{\omega \in[\theta \widetilde{r}, \tilde{r}]}\left[f\left(\omega(s)+\beta_{N}\right)-a(t) \beta_{N}\right]<\widetilde{r}=\|\omega\| .
\end{aligned}
$$

For $\omega \in \partial K_{\bar{r}}$, we have

$$
\begin{aligned}
\|T \omega\| & =\max _{t \in[0, T]} T \omega(t)=\max _{t \in[0, T]} \int_{0}^{T} G(t, s)\left[f\left(\omega(s)+\beta_{N}\right)-a(t) \beta_{N}\right] d s \\
& \geq m T L\|\omega\|>\|\omega\| .
\end{aligned}
$$


From $\left(f_{5}\right)$ and the Arzela-Ascoli theorem it follows that $T$ is completely continuous on $\overline{K_{\widetilde{r}}} \backslash K_{\bar{r}}$. Therefore, by Lemma 2.1, (3.1) has at least a positive solution $\omega(t) \in \overline{K_{\widetilde{r}}} \backslash K_{\bar{r}}$. Namely, (1.3) has at least a positive solution $x(t)=\omega(t)+\beta_{N}$ satisfying $\|x(t)\|<\beta_{N}+\widetilde{r}=\bar{R}$.

Corollary 3.5 Assume that $(\mathrm{H})$ and $(\mathrm{A})$ hold. In addition,

$\left(f_{1}\right) f(\cdot)$ is continuous on $\left(\beta_{N},+\infty\right)$ and $f(x) \geq a^{*} \beta_{N}$ on $\left(\beta_{N},+\infty\right)$. If $f^{\infty}=0$, then (1.3) has at least one positive periodic solution.

Proof If $\left(f_{1}\right)$ holds, then $F(\omega) \geq$ and (i) of (C) hold. From $\left(f_{1}\right)$ and $f^{\infty}=0$ it follows that $\lim _{\omega \rightarrow+\infty} \frac{F(\omega)}{\omega}=0$. Then there exists sufficiently large $R>0$ such that $F(\omega) \leq \epsilon \omega$ for $\omega \geq R$, where $\epsilon M T<1$. Choose $\tilde{r}>\max \left\{\frac{R}{\theta}, \bar{r}\right\}$, where $\bar{r}$ is given in $\left(a_{3}\right)$. Then, for any $\omega \in \partial K_{\tilde{r}}$, one has $\omega(t) \geq \theta\|\omega\|>R$, and further we have

$$
\|T \omega\|=\max _{t \in[0, T]} \int_{0}^{T} G(t, s) F(\omega(s)) d s \leq \epsilon M T\|\omega\|<\|\omega\| .
$$

Combining Lemma 3.4 and Lemma 2.1, we have that (1.3) has at least one positive periodic solution.

Lemma 3.6 Assume that (H), (A), and (E) hold. If $f^{\infty}=+\infty$, then (1.3) has at least two positive periodic solutions.

Proof It is easy to see that (E) implies (C). Then, from Lemma 3.3, we can obtain a solution $x_{1}(t)$ satisfying $\left\|x_{1}(t)\right\|<\bar{R}$.

For any $x(t) \in \partial K_{\hat{R}}$, we get

$$
\begin{aligned}
\|A x\| & =\max _{t \in[0, T]} A x(t)=\max _{t \in[0, T]} \int_{0}^{T} G(t, s) f(x(s)) d s \\
& \leq M T \max _{x \in[\theta \hat{R}, \hat{R}]} f(x(s))<\hat{R}=\|x\| .
\end{aligned}
$$

By the definition of $f^{\infty}=\infty$, there exists $\widetilde{R}>\hat{R}$ such that $f(x) \geq \mu x$ for $x \geq \widetilde{R}$, where $\mu$ satisfies $\mu m T \theta>1$.

Choose $R=\frac{\widetilde{R}}{\theta}$. Then, for $x \in \partial K_{R}$, one has $x(t) \geq \theta\|x\| \geq \widetilde{R}$, and

$$
\begin{aligned}
\|A x\| & =\max _{t \in[0, T]} \int_{0}^{T} G(t, s) f(x(s)) d s \\
& \geq \mu m T \theta\|x\|>\|x\| .
\end{aligned}
$$

From (A) and the Arzela-Ascoli theorem it follows that $A$ is completely continuous on $\overline{K_{R}} \backslash K_{\hat{R}}$. Therefore, by Lemma 2.1, (1.3) has another positive solution $x_{2}(t) \in \overline{K_{R}} \backslash K_{\hat{R}}$.

Finally, we are studying the existence of solutions of (1.3) on $\left(\beta_{i}, \beta_{i+1}\right), i=1,2 \ldots, N-1$.

Lemma 3.7 Assume that (H), (A), and (B) hold. Then (1.3) has at least two positive periodic solutions. 
Proof Let $\omega=u-\beta_{i}$. Then (1.3) is equivalent to the problem

$$
\left\{\begin{array}{l}
\ddot{\omega}+a(t) \omega=f\left(\omega+\beta_{i}\right)-a(t) \beta_{i}=F_{i}(\omega) \\
\omega(0)=\omega(T), \quad \dot{\omega}(0)=\dot{\omega}(T)
\end{array}\right.
$$

From (A) and (B), it follows that

$\left(F_{1}\right) F_{i}(\cdot)$ is nonnegative and continuous on $\left(0, \beta_{i+1}-\beta_{i}\right)$;

$\left(F_{2}\right) F_{i}(\cdot)$ is singular at $x=0^{+},\left(\beta_{i+1}-\beta_{i}\right)^{-}$;

$\left(F_{3}\right)$ Let $\bar{r}_{i}=\tilde{R}_{i}-\beta_{i} \in\left(0, \beta_{i+1}-\beta_{i}\right)$ such that $F_{i}^{*}\left(\bar{r}_{i}\right)<\frac{\bar{r}_{i}}{M T}$.

Then from Lemma 3.3 it follows that (1.3) has at least two positive periodic solutions.

Now we give the proof of the main results.

Proof of Theorem 1.1 The number of the interval $\left(\beta_{i}, \beta_{i+1}\right)$ is $N-1$, then we can obtain the result from Lemma 3.7.

Proof of Theorem 1.2 From Lemmas 3.4 and 3.7 the result follows.

Proof of Theorem 1.3 From Lemmas 3.1 and 3.7 the result follows.

Proof of Corollary 1.4 From Lemmas 3.2 and 3.7 the result follows.

Proof of Theorem 1.5 From Lemmas 3.3 and 3.7 the result follows.

Proof of Theorem 1.6 From Lemmas 3.1, 3.4, and 3.7 the result follows.

Proof of Theorem 1.7 . From Lemmas 3.6 and 3.7 the result follows.

Proof of Theorem 1.8 From Lemmas 3.3, 3.4, and 3.7 the result follows.

Proof of Theorem 1.9 From Lemmas 3.1, 3.6, and 3.7 the result follows.

Proof of Theorem 1.10 From Lemmas 3.3, 3.6, and 3.7 the result follows.

Now we just give an example to illustrate Theorem 1.10.

Example For convenience, we consider the following periodic boundary value problem:

$$
\left\{\begin{array}{l}
\ddot{x}+\mu x=f(x), \\
x(0)=x(T), \quad \dot{x}(0)=\dot{x}(T),
\end{array}\right.
$$

where $a(t)=\mu$ is a constant such that $(\mathrm{H})$ holds, and $f(x)$ is expressed by

$$
f(x)= \begin{cases}C_{01} x^{\nu_{01}}+\frac{C_{02}}{\left(x-\beta_{1}\right)^{v_{02}}}, & x \in\left(0, \beta_{1}\right) \\ \frac{C_{11}}{\left(x-\beta_{1}\right)^{v_{11}}}+\frac{C_{12}}{\left(x-\beta_{2}\right)^{v_{12}}}+\beta_{1} \mu, & x \in\left(\beta_{1}, \beta_{2}\right) \\ \frac{C_{21}}{\left(x-\beta_{2}\right)^{v_{21}}}+\frac{C_{22}}{\left(x-\beta_{3}\right)^{v_{22} 2}}+\beta_{2} \mu, & x \in\left(\beta_{2}, \beta_{3}\right) \\ \frac{C_{31}}{\left(x-\beta_{3}\right)^{v^{31}}}+C_{32} x^{\nu_{32}}+\beta_{3} \mu, & x \in\left(\beta_{3},+\infty\right),\end{cases}
$$


where

(I) $C_{i j}$ are positive constants; $(i=0,1,2,3, j=1,2)$;

(II) $a^{*}=a_{*}=\mu$;

(III) $v_{01}<1, v_{11}, v_{21}, v_{31}>0, v_{32}>1, v_{02}, v_{12}, v_{22}$ are even.

It is obvious that (A) holds. First we show that assumption (B) holds.

(i) $f(x)-a^{*} \beta_{i} \geq 0$ obviously holds for $x \in\left(\beta_{i}, \beta_{i+1}\right)$,

(ii) $\max _{x \in\left[\theta \bar{R}_{i}+(1-\theta) \beta_{i}, \bar{R}_{i}\right]} f(x)<\frac{\bar{R}_{i}-\beta_{i}}{M T}+a_{*} \beta_{i}$

业 $\max _{x \in\left[\theta \bar{R}_{i}+(1-\theta) \beta_{i}, \overline{\bar{R}}_{i}\right]}\left[\frac{C_{i 1}}{\left(x-\beta_{i}\right)^{v_{i 1}}}+\frac{C_{i 2}}{\left(x-\beta_{i+1}\right)^{v_{i 2}}}\right]<\frac{\bar{R}_{i}-\beta_{i}}{M T}$.

If $C_{i j}(i=1,2, j=1,2)$ is sufficiently small, there exists $\tilde{R}_{i} \in\left(\beta_{i}, \beta_{i+1}\right)$ such that the above inequality holds.

Second, if $C_{0 j}(j=1,2)$ is sufficiently small, there exists $\bar{r} \in\left(0, \beta_{1}\right)$ such that $f^{*}(\bar{r})<\frac{\bar{r}}{M T}$, namely (D) holds.

Third, it is clear that $f(x)-a^{*} \beta_{N} \geq 0$ for $x \in\left(\beta_{N}, \hat{R}\right)$. In addition, if $C_{3 j}(j=1,2)$ is sufficiently small, there exists $\hat{R}>\frac{\beta_{N}}{\theta}$ such that

$$
\max _{x \in[\theta \hat{R}, \hat{R}]} f(x)<\frac{\bar{R}}{M T}+\min \left\{a_{*}-\frac{1}{M T}, 0\right\} \beta_{N},
$$

namely (E) holds.

Finally, we can verify that

$$
\begin{aligned}
& f^{0}=\lim _{x \rightarrow 0} \frac{f(x)}{x}=\lim _{x \rightarrow 0} \frac{C_{01} x^{\nu_{01}}+\frac{C_{02}}{\left(x-\beta_{1}\right)^{\nu_{02}}}}{x}=+\infty, \\
& f^{\infty}=\lim _{x \rightarrow \infty} \frac{f(x)}{x}=\lim _{x \rightarrow \infty} \frac{\frac{C_{31}}{\left(x-\beta_{3}\right)^{\nu_{311}}}+C_{32} x^{\nu_{32}}+\beta_{3} \mu}{x}=+\infty .
\end{aligned}
$$

Therefore, by Theorem 1.10, (3.3) has at least $(2 N+2)$ positive periodic solutions.

\section{Conclusion}

Some sufficient conditions are given to illustrate the existence and multiplicity of positive periodic solutions for the second order differential equations

$$
\left\{\begin{array}{l}
\ddot{x}+a(t) x=f(x), \\
x(0)=x(T), \quad \dot{x}(0)=\dot{x}(T) .
\end{array}\right.
$$

For given nonnegative constants $0<\beta_{1}<\beta_{2}<\cdots<\beta_{N}$, the function $f$ may be singular at $x=\beta_{i}$. 


\section{Funding}

The authors were supported by NNSF of China (No.11501165), the projects of Guizhou Provincial Science and Technology Fund (QKH-JICHU [2017], Grant no. 1408, QKH-LH [2014], Grant no. 7366).

\section{Availability of data and materials}

Not applicable.

\section{Competing interests}

The authors declare that they have no competing interests.

\section{Authors' contributions}

All the authors contributed equally and significantly in writing this article. All the authors read and approved the final manuscript.

\section{Author details}

${ }^{1}$ School of Mechanical Engineering, Guizhou Institute of Technology, Guiyang, P.R. China. ${ }^{2}$ College of Science, China pharmaceutical University, Nanjing, P.R. China.

\section{Publisher's Note}

Springer Nature remains neutral with regard to jurisdictional claims in published maps and institutional affiliations.

Received: 3 April 2018 Accepted: 11 July 2018 Published online: 20 July 2018

\section{References}

1. Agarwal, R.P., O'Regan, D., Wong, P.J.Y.: Existence of constant-sign solutions to a system of difference equations: the semipositone and singular case. J. Differ. Equ. Appl. 11, 151-171 (2005)

2. Bonheure, D., De Coster, C.: Forced singular oscillators and the method of lower and upper solutions. Topol. Methods Nonlinear Anal. 22, 297-317 (2003)

3. Chhetri, M., Drábek, P., Shivaji, R.: Analysis of positive solutions for classes of quasilinear singular problems on exterior domains. Adv. Nonlinear Anal. 6, 447-459 (2017)

4. Chu, J., Torres, P.J., Zhang, M.: Periodic solutions of second order non-autonomous singular dynamical systems. J. Differ. Equ. 239, 196-212 (2007)

5. Dunninger, D., Wang, H.: Existence and multiplicity of positive radial solutions for elliptic systems. Nonlinear Anal. 29, 1051-1060 (1997)

6. Franco, D., Torres, P.J.: Periodic solutions of singular systems without the strong force condition. Proc. Am. Math. Soc. 136, 1229-1236 (2008)

7. Franco, D., Webb, J.R.L.: Collisionless orbits of singular and nonsingular dynamical systems. Discrete Contin. Dyn. Syst. $15,747-757(2006)$

8. Ghergu, M., Radulescu, V.: Existence and nonexistence of entire solutions to the logistic differential equation. Abstr. Appl. Anal. 17, 995-1003 (2003)

9. Guo, D., Lakshmikantham, V.: Nonlinear Problems in Abstract Cones. Academic Press, New York (1988)

10. Hakl, R., Torres, P.J., Zamora, M.: Periodic solutions of singular second order differential equations: upper and lower functions. Nonlinear Anal. 74, 7078-7093 (2011)

11. Jiang, D., Chu, J., Zhang, M.: Multiplicity of positive periodic solutions to superlinear repulsive singular equations. J. Differ. Equ. 211, 282-302 (2005)

12. Kaufmann, U., Medri, I.: One-dimensional singular problems involving the $\mathrm{p}$-Laplacian and nonlinearities indefinite in sign. Adv. Nonlinear Anal. 5, 251-259 (2016)

13. Nursultanov, M., Rozenblum, G.: Eigenvalue asymptotics for the Sturm-Liouville operator with potential having a strong local negative singularity. Opusc. Math. 37, 109-139 (2017)

14. Precup, R.: A vector version of Krasnosel'skii's fixed point theorem in cones and positive periodic solutions of nonlinear systems. J. Fixed Point Theory Appl. 2, 141-151 (2007)

15. Rachunková, l., Tvrdý, M., Vrkoč, l.: Existence of nonnegative and nonpositive solutions for second order periodic boundary value problems. J. Differ. Equ. 176, 445-469 (2001)

16. Radulescu, V.D.: Singular phenomena in nonlinear elliptic problems: from blow-up boundary solutions to equations with singular nonlinearities. In: Handbook of Differential Equations: Stationary Partial Differential Equations, vol. 4, pp. 485-593. North-Holland, Amsterdam (2007)

17. Torres, P.J.: Existence of one-signed periodic solutions of some second-order differential equations via a Krasnoselskii fixed point theorem. J. Differ. Equ. 190, 643-662 (2003)

18. Torres, P.J.: Non-collision periodic solutions of forced dynamical systems with weak singularities. Discrete Contin. Dyn Syst. 11, 693-698 (2004)

19. Torres, P.J.: Weak singularities may help periodic solutions to exist. J. Differ. Equ. 232, 277-284 (2007)

20. Wang, F., Chu, J., Siegmund, S.: Periodic solutions for second order singular differential systems with parameters. Topol. Methods Nonlinear Anal. 46, 549-562 (2015)

21. Wang, H.: Positive radial solutions for quasilinear systems in an annulus. Nonlinear Anal. 63(5-7), 495-2501 (2005)

22. Wang, H.: Positive periodic solutions of singular systems with a parameter. J. Differ. Equ. 249, 2986-3002 (2010)

23. Yan, P., Zhang, M.: Higher order nonresonance for differential equations with singularities. Math. Methods Appl. Sci. 26, 1067-1074 (2003)

24. Zhang, M.: Periodic solutions of equations of Ermakov-Pinney type. Adv. Nonlinear Stud. 6, 57-67 (2006) 